\title{
Matar, dejar morir y eutanasia en el proyecto de ley sobre derechos de las personas y en la doctrina chilena
}

\author{
RODOLFO FIGUEROA G. ${ }^{a}$
}

\section{To kill, let die and euthanasia in the bill of rights of patients and in the Chilean doctrine}

The Bill of Rights for Patients provides the patient with autonomy for disposing of his life, enabling him to reject those treatments that unnecessarily prolong his life. However, the bill does not allow an artificial acceleration of death. Therefore, the bill does not permit euthanasia (at least, certain form of it) nor assisted-suicide. However, according to the practice of medicine and also Chilean doctrine, it is permitted to inject morphine to a patient to relieve his pain, even though that could hasten his death. In consequence, it is allowed for the patient to dispose of his life and also to inject in him morphine for pain relief, endangering his life, but neither euthanasia nor assisted-suicide is allowed. Is this coherent? According to Chilean doctrine, it could be coherent under the condition of accepting the distinction between killing and letting die and also the double effect doctrine. The problem is that there is abundant English literature in the realm of moral philosophy to disregard both conditions. Therefore, it is possible to claim that the Bill is not coherent and that the Chilean doctrine is based upon a distinction and a doctrine that are not acceptable.

(Rev Med Chile 2011; 139: 655-659).

Key words: Ethics, medical; Euthanasia; Suicide, assisted.

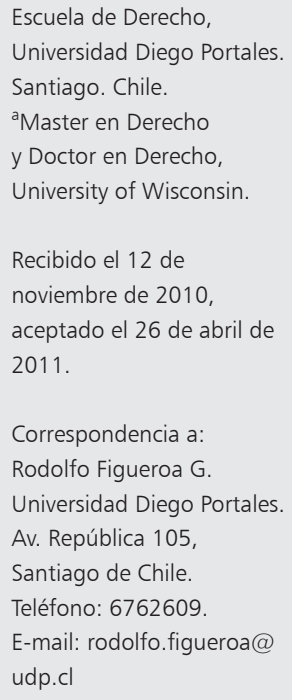

su sufrim stá justificado desconectar a un paciente terminal? ¿Está permitido suministrarle una dosis de opiáceos destinada a aliviar su muerte? ¿Está permitida la eutanasia? Estos son problemas de bioética, que se relacionan con derechos de autonomía de los pacientes.

Actualmente, existe en el Congreso Nacional un proyecto de ley que regula los derechos y deberes que los pacientes, Mensaje no 223-354, Boletín 4398-11, de 2006. Este proyecto confiere autonomía a las personas para disponer de su vida en ciertos casos, pero no permite la eutanasia ni la asistencia al suicidio. Así, la primera pregunta con que comenzamos se respondería afirmativamente; también la segunda, pero no la tercera. ¿Por qué? Según la doctrina nacional en bioética, estos casos se deben tratar de modo distinto por dos razones: primero, por la diferencia entre matar y dejar morir; segundo, por la doctrina del doble efecto. El objetivo de este trabajo es cuestionar ambas razones: recurriendo a literatura anglosajona en materia de bioética y filosofía moral se intenta demostrar que es inadmisible la distinción entre matar y dejar morir para los casos en que se propone y que la doctrina del doble efecto es inútil para distinguir qué casos se deben permitir y cuáles se deben prohibir.

\section{Desarrollo}

\section{Derechos de autonomía de los pacientes en el proyecto de ley (Boletín 4398-11) \\ El proyecto de ley reconoce varios derechos de autonomía, pero el que interesa acá comprende}


dos situaciones: i) El derecho de toda persona "a otorgar o denegar su voluntad para someterse a cualquier procedimiento o tratamiento vinculado a su atención de salud..." (artículo 14, Boletín 4398-11) y ii) El derecho del enfermo terminal "a otorgar o denegar su voluntad para someterse a cualquier tratamiento que tenga como efecto prolongar artificialmente su vida." (artículo 17, Boletín 4398-11).

Como se puede advertir, este es un derecho de autonomía porque el paciente tiene libertad para adoptar una decisión que incide en su propio destino; no está obligado a someterse a tratamientos médicos que no desee, ni tampoco a continuar con ellos, incluyendo aquellos que artificialmente prolonguen su vida. Por tanto, el paciente puede escoger un curso de acción que implique morirse. Sin embargo, el proyecto establece límites a este derecho, entre ellos, el del artículo 17: "En ningún caso, el rechazo de tratamiento podrá implicar como objetivo la aceleración artificial del proceso de muerte."

Esta limitación excluye el suicidio asistido. ¿Excluye también la eutanasia? Depende de cómo se aplique el concepto de eutanasia a casos concretos. Por ejemplo, para Goic ${ }^{1}, \mathrm{Beca}^{2}$, Ugarte ${ }^{3}$, Rodríguez $z^{4}$, Vivanco ${ }^{5}$, entre otros, desconectar a un paciente de un ventilador mecánico o retirar medios extraordinarios no constituye eutanasia. Por tanto, para esos autores el proyecto de ley excluye la eutanasia. Sin embargo, Foot $^{6}$ y Gruzal$\mathrm{ski}^{7}$ sostienen que la desconexión es una forma de eutanasia pasiva. Más aún, Hopkins afirma que la desconexión corresponde derechamente a eutanasia activa, pues desconectar es un hacer, no un omitir. ${ }^{8}$ Por tanto, si la desconexión es una forma de eutanasia, el proyecto no prohíbe toda forma de eutanasia sino sólo algunas (por ejemplo, inyectar una droga con el propósito de causar la muerte).

\section{Hipótesis de muerte}

Para analizar el derecho de autonomía y su límite me voy a valer de un esquema de Thomson ${ }^{9}$ que distingue cuatro casos: 1) Desconexión; 2) No conexión; 3) Proporcionar droga letal y 4) Inyectar droga letal. Thomson llama droga letal a aquella que en un contexto dado permite producir la muerte.

Aplicando este esquema al proyecto de ley, tendríamos lo siguiente:

a) El derecho del paciente a denegar su volun- tad para someterse a cualquier tratamiento médico permitiría los casos 1) y 2) (suponiendo que el derecho a denegar la voluntad para someterse a un tratamiento médico incluye descontinuar un tratamiento en curso).

b) El límite de no acelerar artificialmente el proceso de muerte prohibiría los casos 3) y 4).

c) Junto con lo anterior, es necesario considerar una quinta hipótesis: 5) proporcionar morfina a un paciente terminal para aliviar su dolor, aun pudiendo prever que esa dosis pueda provocar una depresión del sistema respiratorio que conduzca a la muerte. Hay que decir que existe literatura inglesa que descarta que este caso pueda ocurrir ${ }^{10}$, también chilena ${ }^{11}$, y que señala que el problema real es la sedación hasta la inconsciencia, como cree Cellarius ${ }^{12}$ y Arratia. ${ }^{11}$ Sin embargo, este caso 5) es una hipótesis validada por la doctrina nacional: los citados Goic, Beca, Vivanco y Rodríguez, y también Taboada ${ }^{13}$, Misseroni ${ }^{14}$ y Twycross ${ }^{15}$, de modo que voy a considerar este caso 5 ).

Pues bien, el paciente tiene derecho a solicitar alivio en la hipótesis del caso 5). Por tanto, debe estimarse como un caso permitido.

En suma, se pueden estimar permitidos los casos 1), 2) y 5) y prohibidos los casos 3 ) y 4).

La pregunta que cabe formular es: ¿Es coherente hacer eso?

Sostengo que no lo es por las siguientes consideraciones:

i) Los casos 1) y 2) se permiten sobre la base del principio de autonomía. Pues bien, dicho principio también justifica la permisión de los casos 3) y 4). Prohibir estos casos es negar la autonomía, lo que produce incoherencia.

ii) Los casos 3) y 4) se prohíben en Chile básicamente por la convicción de que la vida es un bien indisponible, como creen, entre otros, Arratia ${ }^{11} y$ Vivanco $^{16}$, aunque también existen otras razones. ${ }^{1}$ Pues bien, si consideramos el argumento de que la vida es un bien indisponible, no podrían permitirse los casos 1) y 2) cuando en ellos se pueda prever que se producirá la muerte. Sin embargo, esos casos se permiten, lo que es incoherente.

iii) Si consideramos que la desconexión constituye una forma de eutanasia, entonces el proyecto de ley permite cierta forma de eutanasia (caso 1) y prohíbe otra (caso 4). ¿Por qué una sí y la otra no?

iv) El caso 5), si efectivamente se produce la muerte, se parece al caso 4) pues se inyecta al paciente algo que se puede prever es condición sine 
qua non de la muerte si ella se produce. Pues bien, es incoherente prohibir uno y permitir el otro.

Como se puede advertir, hay varias incoherencias en el proyecto de ley. La doctrina nacional cree que no, tal como se explica en la sección siguiente.

\section{Matar y dejar morir y la doctrina del doble efecto}

Revisando la literatura chilena sobre bioética referida a eutanasia y suicidio asistido, parece bastante nítido concluir que para varios autores nacionales no habría problema ni incoherencia alguna en permitir los casos 1), 2) y 5) y prohibir los casos 3) y 4) básicamente por dos razones: 1) Por la distinción entre matar y dejar morir y 2) En virtud de la doctrina del doble efecto (DDE). Veremos estas dos situaciones.

\subsection{Matar y dejar morir (DDA)}

La distinción entre matar y dejar morir sirve para justificar la permisión de los casos 1) y 2) y la prohibición de los casos 3) y 4). Esta distinción es conocida en el ámbito anglosajón como la doctrina del hacer y permitir (Doctrine of Doing and Allowing, la DDA) y es apoyada explícitamente por la doctrina nacional: Moran $^{17}$, Rodríguez ${ }^{4}$, Beca $^{2}$, Vivanco. ${ }^{5}$ Los casos 1) y 2) serían casos en que se omite algo: se omite conectar (no conexión) o se omite continuar con un tratamiento (desconexión). En esos casos, se deja que la muerte ocurra. El paciente muere de la enfermedad o condición de salud en que se encontraba. La conducta del médico no es causa de la muerte. En cambio, en los casos 3 ) y 4) se hace algo para producir la muerte: el médico proporciona una droga que es capaz de producir la muerte (caso 3 ) al paciente para que este se suicide, o el médico mata al paciente inyectándole esa droga a su requerimiento (caso 4 ).

Así, los casos 1) y 2) se pueden permitir porque no se mata al paciente sino que se deja que la muerte ocurra. En cambio, se debe prohibir el caso 4) porque se mata al paciente y el caso 3 ) porque se contribuye a que se mate.

En consecuencia, la diferencia entre matar y dejar morir es utilizada por la doctrina para justificar la permisión de 1) y 2) y la prohibición de 3) y 4).

Finalmente, y esto es obvio, quienes distinguen entre matar y dejar morir creen que existe una diferencia moral relevante entre ambos, en el sentido de que lo primero no es tolerable en tanto lo segundo sí.

\subsection{La doctrina del doble efecto (DDE)}

La DDE sirve para justificar la permisión del caso 5) y la prohibición del 4).

Esta es una doctrina originalmente católica ${ }^{18}$ diseñada para determinar la corrección o incorrección moral de una conducta en situaciones en que se causará un daño a alguien. En términos generales, siempre es moralmente incorrecto ejecutar intencionalmente un acto malo con el objetivo de producir consecuencias buenas, pero puede ser permisible moralmente ejecutar un acto bueno aunque pueda producir consecuencias malas.

Esta doctrina distingue entre desear y prever ${ }^{19}$ y se apoya en la DDA. Una conducta puede tener dos efectos y es permisible si el efecto deseado es bueno y compensa a otro malo que no es deseado sino meramente previsto 9 . La DDE posee varios requisitos. El que importa aquí está relacionado con las intenciones de los médicos. En el caso 5), la intención del médico es aliviar el sufrimiento y un posible efecto secundario no querido es la muerte del paciente. Dado que un fin central de la medicina es aliviar el sufrimiento, está justificado inyectar morfina aunque sea previsible (pero no querida) la muerte del paciente por depresión del sistema respiratorio. En cambio, en el caso 4), la intención del médico es matar al paciente. No hay un efecto bueno y uno malo. Por eso, no es justificable. Esta doctrina es seguida prácticamente todos los autores nacionales que han escrito sobre este tema, ya citados.

En conclusión, y para cerrar esta parte: conforme la literatura chilena, sería coherente permitir los casos 1), 2) y 5) y prohibir los casos 3) y 4) a condición de que sean válidas la DDA y la DDE. Esto se objetará en la sección siguiente.

\section{Objeciones a la DDA y la DDE}

Existe abundante literatura inglesa en el ámbito de la filosofía moral para estimar que las condiciones explicadas en la sección precedente son discutibles y, por tanto, no permiten justificar un trato distinto entre los casos 1), 2) y 5) y los casos 3) y 4). Veámoslo:

\subsection{La supuesta diferencia entre matar y dejar morir (DDA)}

Rachels $^{20}$, Foot ${ }^{6}$, Gruzalski ${ }^{21}$, Hopkins ${ }^{8}$ y Dwor$\operatorname{kin}^{22}$ señalan que no existe ninguna diferencia moral relevante entre matar y dejar morir en un mismo contexto causal. Asfixiar a un recién na- 
cido o dejarlo morir de hambre no se distinguen en términos morales. Si mi enemigo ingresa a mi pieza de hospital para liquidarme, da lo mismo, moralmente, que me dispare o desconecte mi respirador ${ }^{9}$. Rachels pone el ejemplo de la bañera: una persona quiere heredar a su sobrino y decide ahogarlo cuando se esté dando un baño. Da lo mismo moralmente ahogar al niño que, al ingresar al baño, dejarlo morir al descubrir que se está ahogando solo ${ }^{20}$. Lo que señalan estos autores es que no es menos malo dejar morir que matar, como sí cree la doctrina chilena.

Thomson objeta aquella postura que sostiene que cuando se desconecta al paciente simplemente se deja que las cosas sigan su curso, de modo que el paciente muere de la condición de salud subyacente. Esta autora señala que si remuevo la viga que sostiene el techo y el derrumbe mata las personas que estaban debajo, no puedo decir que no los maté; no puedo alegar que dejé a la ley de gravedad seguir su curso'. Remover la viga es un factor causal necesario, así como lo es remover (desconectar) el sistema de soporte vital del paciente; es decir, sin ellos, la muerte no se habría producido en ese momento.

Ahora bien, hay autores que sí aceptan diferencia moral entre matar y dejar morir, pero sólo para las denominadas hipótesis de vidas en conflicto, no para los denominados casos de comparación. Casos de vidas en conflicto son aquellos en que varias personas están en una situación en virtud de la cual algunas de ellas necesariamente morirán; quienes mueran dependerá de una acción o una omisión. Hay muchos ejemplos en la filosofía moral, como el caso del Trolley ${ }^{23}$, o la máquina de Malm: John y Mary están atrapados en una máquina. Si aprieto un botón, la máquina libera a John y Mary muere. Si no hago nada, la máquina mata a John y libera a Mary. Cual muera dependerá de si ejecuto una acción (mato) o una omisión (dejo morir). En estos casos es relevante distinguir entre matar y dejar morir. Si diera lo mismo la acción o la omisión, entonces el agente tendría libertad para decidir quien vive y quien muere, trasladando la muerte de John a Mary. Sin embargo, no existe un principio moral que conceda esa libertad al agente. Por eso no da igual que omita o apriete el botón ${ }^{24}$.

En cambio, en casos de comparación, como el de la bañera, o dejar al niño morir de hambre, o los casos 1) al 5) ya explicados, no es relevante la DDA pues no se trata de casos en que haya varias personas en peligro en términos tales que quien viva o muera dependa de una acción u omisión. Ambas conductas producen el mismo resultado.

Por tanto, existe literatura relevante en materia de filosofía moral y bioética que permite cuestionar el uso de la distinción entre matar y dejar morir para justificar la permisión de los casos 1) y 2) y la prohibición de los casos 3 ) y 4).

\subsection{La doctrina del doble efecto (DDE)}

A pesar de que la DDE tiene bastante aceptación en la literatura chilena, existe considerable doctrina en la filosofía moral inglesa que objeta su utilidad. Hart señala que entre matar intencionalmente (prohibido por la DDE) y causar la muerte habiéndolo previsto pero no querido (permitido por la DDE) sólo existe diferencia en la estructura causal y diferencia verbal, pero esas diferencias no se relacionan con factores morales y no son justificables en ninguna teoría de la moralidad..$^{18}$ Muchos autores consideran la DDE intrínsecamente confusa y, por tanto, inservible: Foot $^{23}$, Thomson ${ }^{9}$, Davis ${ }^{25}$, McIntyre ${ }^{26}$, Scanlon ${ }^{27}$, Kamm. ${ }^{28}$ Por ejemplo, Thomson advierte cinco problemas; uno de ellos es el siguiente: es absurdo que la permisibilidad de la conducta dependa de la intención del médico. ¿Se puede desconectar un paciente? (caso 1). La DDE dice que depende de las intenciones del médico; si pretende la muerte, la respuesta es negativa, lo que es un problema porque se ha asumido que el caso 1) se permite siempre. ¿Se puede inyectar morfina? (caso 5) Según la DDE, depende: un doctor que inyecta morfina pero no para aliviar el sufrimiento sino porque quiere que el paciente muera, actuaría de modo moralmente incorrecto; en cambio otro que lo hiciera para aliviar el sufrimiento, actuaría bien moralmente. Por tanto, el caso 5) no estaría siempre permitido, sino que habría que indagar en las intenciones del médico ${ }^{9}$. A algunos médicos les permitiríamos 1) y 5) y a otros médicos no, dependiendo de sus intenciones. Esta conclusión no es compatible con la doctrina nacional, para la cual la DDE siempre justifica el caso 5).

Por tanto, existe abundante literatura para dudar que la DDE sea una herramienta conceptual útil para justificar la permisión del caso 5) y la prohibición del 4).

En síntesis, existe literatura relevante en el campo de la bioética y la filosofía moral-aparentemente ignorada en Chile-que permite cuestionar 
las dos condiciones que la doctrina nacional utiliza (la DDA y la DDE) para justificar un trato distinto entre los casos 1), 2) y 5) y los casos 3) y 4).

\section{Conclusiones}

El proyecto de ley de derechos de los pacientes permite los casos 1), 2) y 5) pero prohíbe los casos 3) y 4).

Conforme la doctrina nacional, esto no es incoherente a condición de que aceptemos que existe diferencia moral relevante entre matar y dejar morir, y aceptemos, además, la doctrina del doble efecto.

Sin embargo, existe abundante literatura inglesa en el campo de la filosofía moral según la cual estas condiciones no son aceptables.

En consecuencia, el proyecto de ley es incoherente y la doctrina nacional en bioética apoya dos doctrinas (DDA y DDE) que son ampliamente cuestionables si se aplican a los casos 1) al 5).

\section{Referencias}

1. Goic A. Apuntes sobre la Eutanasia. Rev Med Chile 2005; 133: 371-5.

2. Beca JP, Ortiz A, Solar S. Derecho a Morir. Rev Med Chile 2005; 133: 601-6.

3. Ugarte JJ. El derecho a la vida y la Constitución. Rev Chil Derecho 2006; 33: 509-27.

4. Rodríguez E. La eutanasia y sus argumentos. Ars Medica 2000; 2: 45-57.

5. Vivanco A. La eutanasia ante el derecho. Ars Medica 2006; 12: 53-92.

6. Foot P. Euthanasia. Philos Public Aff 1977; 6: 85-112.

7. Gruzalski B. Killing by Letting Die. Mind 1981; 90: 91-8.

8. Hopkins PD. Why does removing machines count as "passive" euthanasia? Hastings Cent Rep 1997; 27: 29-37.
9. Thomson JJ. Physician-Assisted Suicide: Two Moral Arguments. Ethics 1999; 109: 497-518.

10. Skyes N. Thorns A. The use of opiods and sedatives at the end of life. Lancet Oncol 2003; 4: 312-8.

11. León F, Arratia A. El debate actual sobre la legalización de la eutanasia. Ars Medica 2006; 12: 39-51.

12. Cellarius V. Terminal Sedation and the imminence condition. J Med Ethics 2008; 34: 69-72.

13. Taboada P, Rodríguez A, Vercellino M. Dimensión ética del morir. Ars Medica 2000; 2: 31-44.

14. Misseroni A. Consideraciones jurídicas en torno al concepto de eutanasia. Acta Bioeth 2000; VI: 249-63.

15. Twycross R. Medicina paliativa: filosofía y consideraciones éticas. Acta Bioeth 2000; VI: 29-46.

16. Vivanco A. Bien morir: Análisis crítico de jurisprudencia constitucional y penal. Ars Medica 2000; 2: 83-97.

17. Morán S. Una reflexión sobre la eutanasia. Ars Medica 2001; 5: 115-8.

18. Hart HLA. Punishment and Responsibility: Essays in the Philosophy of Law. Oxford University Press USA; 2008.

19. Foot P. The Problem of Abortion and the Doctrine of the Double Effect. Oxford Rev 1967; 5: 5-15.

20. Rachels J. Active and Passive Euthanasia. N Engl J Med 1975; 292: 78-80.

21. Gruzalski B. Killing by Letting Die. Mind 1981;90:91-98.

22. Dworkin. G. Sex, Suicide, and Doctors, Ethics 1999; 109: 579-85.

23. Foot P. The Problem of Abortion and the Doctrine of the Double Effect. Oxford Rev 1967; 5: 5-15.

24. Malm H. M. Killing, Letting Die, and Simple Conflicts. Philos Public Aff 1989; 18: 238-58.

25. Davis N. The Doctrine of Double Effect: Problems of Interpretation. Pac Philos Quart 1984; 65:107-23.

26. McIntyre A. Doing Away with Double Effect, Ethics 2001; 111: 219-55.

27. Scanlon T. Moral Dimensions. Belknap Press of Harvard University Press England; 2008.

28. Kamm, F.M The Doctrine of Double Effect: Reflexions on Theoretical and Practical Issues. J Med Philos 1991; 16: 571-85. 